\title{
Design of a Web-based LBS Framework Addressing Usability, Cost, and Implementation Constraints
}

\author{
Jacob Delfos ${ }^{\mathrm{a}}$, Tele Tan $^{\mathrm{b}}$, Bert Veenendaal $^{\mathrm{a}}$
}

${ }^{a}$ Department of Spatial Sciences, Curtin University of Technology, GPO Box U1987 Perth, Western Australia 6845.

${ }^{b}$ Department of Computing, Curtin University of Technology, GPO Box U1987 Perth, Western Australia 6845.

\author{
Corresponding Author: \\ Jacob Delfos \\ Email: Jacob.Delfos@postgrad.curtin.edu.au \\ Phone: +61892667562 \\ Fax: +61892662703
}

Keywords: LBS, Positioning, Usability, Adaptivity, User Modelling, User Monitoring

\begin{abstract}
This research investigates barriers that prevent Location Based Services (LBS) from reaching its full potential. The different constraints, including poor usability, lack of positioning support, costs, and integration difficulties are highlighted. A framework was designed incorporating components based on existing and new technologies that could help address the constraints of LBS and increase end-user acceptance. This research proposes that usability constraints can be addressed by adapting a system to user characteristics which are inferred on the basis of captured user context and interaction data. A prototype LBS system was developed to prove the feasibility and benefit of the framework design, demonstrating that constraints of positioning, cost, and integration can be overcome. Volunteers were asked to use the system, and to answer questions in relation to their proficiency and experience. User-feedback showed that the proposed combination of functionality was well-received, and the prototype was appealing to many users. Ground-truths from the survey were related back to data captured with a user monitoring component in order to investigate whether users can be classified according to their context and how they interact. The results have shown that statistically significant relationships exist, and that by using the $\mathrm{C} 4.5$ decision-tree, computer proficiency can be estimated within one class-width in $76.7 \%$ of the cases. These results suggest that it may be possible to build a user-model to estimate computer proficiency on the basis of user-interaction data. The user model could then used to improve usability through adaptive user-specific customisations.
\end{abstract}




\section{Introduction}

Recent years have seen an exciting increase of spatial technology into the lives of the general public. Examples of this are car navigation devices, GPS-enabled mobile phones, and interactive maps embedded into websites. Because of this, the community has a greater awareness and understanding of mapping and positioning tools and information. In parallel to these developments there have been significant advances in other fields that could take advantage of the introduced spatial capabilities. Examples are social networking websites such as Facebook, MySpace, and Twitter [1-3], mobile devices, widespread wireless internet availability, and web-based technology including the introduction of AJAX-based designs [4].

Location Based Services (LBS) provide the user with information or services specific to the location of the user. Given the recent technological advances, it would appear that there is ample opportunity for the emergence of LBS systems that combine newly introduced spatial capabilities with other services. However, despite the general state of technology, LBS systems are not as commonplace as they could be. There is ample research on the benefits and potential of LBS systems [5, 6], but there are comparatively few implementations and published designs. Many established LBS applications are aimed at mobile phone users, and are restricted to a particular device, such as Nokia Ovi Maps [7], or a particular provider, such as the Uandme-based Whereis Everyone service offered by Telstra [8], and the older MapPoint [9] and Multimap [10] based Finda application offered by Optus in Australia. Other existing LBS applications include the restaurant recommender system Urbanspoon [11] and Google Maps [12] which support basic positioning and the capability to search for services. In addition, Facebook [13] plans to introduce LBS services in April 2010 [14]. What most implementations have in common so far, however, are limitations in scope, accessibility, interoperability, or essential capabilities such as positioning or mapping. Most implementations also fail to address usability issues related to the traditionally specialist nature of spatial systems $[15,16]$.

This research investigates some of the constraints that prevent LBS from reaching its full potential, and presents a framework that attempts to address these constraints, using new and existing methods. The proposed framework integrates the most useful and appealing capabilities available, such as interactive mapping, social interaction, and high-availability positioning, while addressing usability issues through user-specific adaptivity. The result is a user-friendly integrated environment that presents locationbased information in the spatial dimension, in context with the location of the user and their friends. The design is based on low-cost technology, removing an important barrier to implementation costs and user acceptance.

This paper first provides a background on the current state of developments in LBS, and on the constraints that the proposed framework tries to address. Different technologies are presented that can help form solutions to the identified problems. Usability is considered a particularly relevant constraint; this research aims to explore how adaptivity through user monitoring and user modelling could be a viable approach to this problem. The different components that constitute the framework are then described, highlighting how they attempt to address the constraints. This paper also explains how social interaction components could leverage the appeal of an LBS application to the users, based on the popularity of these types of applications. The architecture of a prototype implementation of the proposed LBS framework, called POMICOS (Positioning, Mapping, Information and Communication System), is then presented.

The POMICOS prototype was used to capture user-interaction information, in order to investigate the relationships between how a user interacts with a website, and how proficient they are in the use of it. The results of the data collection and analysis are presented in Section 4, as well as a user-model that could be used to drive a future adaptivity component to improve usability.

\section{Background}

This section describes the current state of research and developments in LBS, and to a degree in geospatial systems in general. It highlights some of the shortcomings in the current literature and implementations, and discusses which methodologies currently available could help to overcome these shortcomings. 


\subsection{Constraints in LBS Systems}

There is much potential for LBS, as the spatial dimension can be applied to almost anything. As such, there is a large amount of literature that expands on the potential applications and suggesting location can improve existing scenarios $[6,17,18]$. However, in practise, there are a number of obstacles that need to be overcome to make these scenarios reality:

- Capturing quality location data - This is clearly a very important bit of information to LBS systems, but not always as easily obtained. There are a number of ways to obtain locations, including WiFi-positioning [19-21], RFID-positioning [22], GSM-positioning, [23], motion detection [24], IP-positioning [25], and GPS [26]. However, most methods lack availability, precision, or both. The large amount of research still being undertaken on positioning methodologies and in particular, their interoperability, suggests that there still remains much opportunity for improvement.

- Infrastructure costs and availability - Which resources are required to make positioning and communication work? Most positioning methods require an infrastructure of some sort, including WiFi, RFID, and GPS. There is often a substantial cost associated with the required infrastructure, and with devices needed by users [24, 27].

- System integration - Many hypothetical scenarios in literature describe a system that performs an action on behalf of a user by communicating seamlessly and autonomously with other systems. An example of this is the booking of a ticket or a restaurant table without user intervention [28]. In reality, communication between heterogeneous systems, such as between an LBS and a ticketing or booking system, is not straightforward [29-31]; not all types of systems have well-defined protocols or common interfaces by which to communicate. The application of appropriate standards is not yet sufficiently widespread to achieve seamless interoperability, preventing these hypothetical scenarios from becoming reality.

- Usability - This concept is defined by the International Standards Organisation (ISO 924111) as "the extent to which a product can be used by specified users to achieve specified goals with effectiveness, efficiency, and satisfaction in a specified context of use" [32]. Usability has been found to be particularly poor for web mapping systems when assessed against ISO standards [33], but geographic information systems (GIS) in general are found to exhibit poor usability [15]. The complexity of GIS have caused it to become accessible to only a handful of sophisticated users, giving it the image of an "elitist" technology [16]. The general public may not possess the spatial literacy required to efficiently operate a spatial software application.

- Contextualisation - Many spatial systems are by nature "all-encompassing", and as such it may not be easy for the user to convey their goals. If a user has to specifically provide information that the system could have worked out on the basis of available contextual information, it could degrade the usability [34].

Williams [25] explains that there is no generic one-size-fits-all solution to LBS, and that each LBS system must be custom developed to a particular situation. Although this may seem a somewhat restrictive view, it is most likely valid as long as problems with standardisation and system heterogeneity are not adequately addressed.

\subsection{Methods to Address Limitations}

A number of methods and technologies exist to address the limitations of LBS, even though some of these were originally perhaps not intended to be applied to LBS. This section describes the methods and research that can potentially be used to address the constraints.

\subsubsection{Improved positioning methods}

The ability to determine the location of a user is very much dependent on the current state of technology. For example, improvements to GPS precision and methods to overcome the line-of-sight 
problem that prevents GPS technology from working indoors or in built-up areas [35]. However, by making smarter use of existing technology, some of the positioning problems could be overcome. For example, fingerprinting is a way to take WiFi-positioning to a precision not possible using ordinary triangulation [22, 23, 36]. Precisions of several metres have been achieved using this method [23]. Fingerprinting can even be expanded upon by including ambient measurements from device sensors, as demonstrated in the SurroundSense project, which uses motion, light and sound to fingerprint relative locations using mobile phones [37]. Accuracy of WiFi-positioning can be enhanced by methods that detect whether access points have moved, although this may require access points being able to measure the signal strength of neighbouring points [38]. Precision in IP-positioning can be significantly improved by methods that take into account the hierarchical nature of networks [25, 39]. Cell phone triangulation is also being used to obtain positions, but this method only offers limited precision of 110-200 metres [20, 23]. WiFi-, IP and GSM-positioning are methods that allow for indoor positioning, unlike GPS. However, GPS can be combined with WiFi to achieve both availability and high-accuracy $[40]$.

\subsubsection{Cost-efficient technology}

A cost-efficient method to implement a system is to make use of resources that already exist, even if they were originally put in place for a different purpose. For this reason, WiFi-positioning is attractive, because it uses the access points of wireless networks as location beacons [21, 40]. Most portable computers are equipped with WiFi adaptors, meaning there is no additional cost to the end-user. WiFi positioning typically offers sufficient precision for LBS applications [20]. IP-address based positioning makes use of information readily available, and does not require the availability of any additional physical devices to work.

Modern browser-based methods and technologies, such as AJAX [4], make it possible and attractive to use browsers as a platform to run almost any application as is evident with the emergence of Web 2.0 applications. Web-based applications remove the need to have custom software installed on client computers, thereby reducing cost and promoting ease of access to a system by users.

The growth of Open-Source software in the geospatial field has seen the emergence of powerful applications [41-44] capable of supporting enterprise-standard systems. It is no longer necessary to rely on proprietary software with significant licensing fees in order to implement an LBS system.

Recent years have seen the emergence of computationally powerful portable devices. This means that the need for purpose-built devices, such as those developed for the FLAME2008 project [27], no longer applies if LBS systems make use of the devices a user already has, such as a mobile phone.

\subsubsection{Standards}

In the past few years, much effort has put towards interoperability and standardisation in the geospatial field. The Open Geospatial Consortium (OGC) is the body responsible for defining and maintaining these standards. As a result, communication and integration between heterogenic geospatial systems has greatly improved. However, LBS often deals with communication between spatial systems and non-spatial systems, such as among restaurant booking systems [28], mobile phone systems [6], or social applications [45]. Not all areas involved have the same level of standardisation, and if they do, these standards may not have been implemented in the systems involved. An OGC OpenLS standard [46] was developed to facilitate communication between different systems in an LBS context. If this standard is widely adopted by the systems involved, it should help to address these interoperability problems.

\subsubsection{Context-sensitivity}

In order to determine the goals or requirements a user may have, all information available about the user, including location, should be taken into account. The location of a user with respect to his/her surroundings can provide us with a context, which may tell us something about the user's background, activity or interests. Likewise, people who surf the internet often unknowingly provide web-servers with information about their context, including their operating system, browser version, screen 
resolution, and other details that give clues regarding their preferences or environment. They also reveal their IP-address, which can be used to resolve their approximate geographic location. User context can be a valuable source of information for geocoding [47].

An example of using IP addresses for identifying location is a research initiative run at Curtin University, where the locations of visitors to numerous websites are determined, and published as pins on a map, along with their (not personally identifiable) platform information. Fig. 1 shows a screenshot of the mapping interface to the logged data.

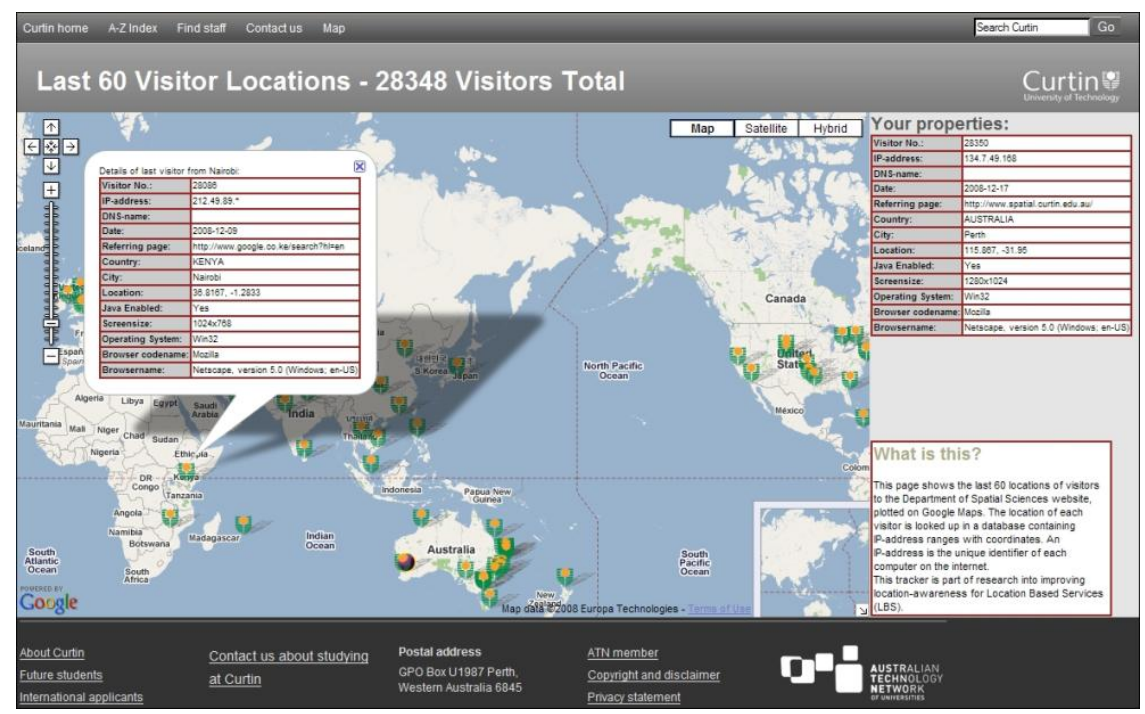

Fig. 1 Screenshot of web-map interface showing visitors to a website of Curtin University [48]

Any information that can be automatically collected about the user should be considered for use in adapting the functionality and customising the information provided, if this can help to better serve the user.

\subsubsection{Usability through adaptivity}

Usability is of particular importance to LBS, as LBS systems are usually developed with the general public, as opposed to expert users, in mind. People with varying interests and different levels of expertise must be able to use the system. Considering the inherent complexity of geospatial systems in general, and the potentially unlimited amount of information an LBS could provide, this is an almost impossible task unless the system adjusts its functionality and content to the characteristics, context, and preferences of the user [6, 27, 28, 49-54]. This kind of behaviour is called "adaptivity". Spatial systems are particularly suited for adaptive content since a focus on the user's geographic region of interest allows for filtering away much irrelevant information. Customisation of information content provided through portals can greatly increase its fitness for use, and hence better meet user requirements [55].

\subsubsection{User modelling}

Adjustments to the content and functionality of a system should be performed on the basis of information known about the user, including, but not limited to, context-related information. Information about a user can be collected through user monitoring, where the interaction of a user with a system is investigated [56-58]. User monitoring includes capturing interface events [58,59] as well as analysing which information the user requests [53, 60]. The information available about the user can then be analysed in order to draw conclusions about the user. The concept of user modelling is about classifying and quantifying different aspects of a user. Different methods exist for building a user model, including stereotyping $[60,61]$ and various methods of machine learning, where user models are built and adjusted on-the-fly [49]. 
The output from a user model can be used to drive adaptive changes to the content and functionality of a system, to better suit individual users [60]. This can be fully automated, but there is reason to believe that usability is improved where users have some control over the adaptation [62].

\section{$3 \quad$ Research Methodology}

This section outlines the objectives, the proposed LBS framework, and how a prototype based on the framework was implemented to address the constraints. It also explains how the prototype was used for data-collection, to gather data for user modelling.

\subsection{Research Objectives}

The purpose of this research is to address some of the constraints of LBS discussed in Section 2.1, using the methodologies explained in Section 2.2. The objective is to propose a framework that incorporates positioning, context-sensitivity, interoperability, user-modelling and adaptivity in a costeffective way. It is expected that if some, or all, of these constraints were addressed, it would significantly improve the feasibility of LBS initiatives as well as the uptake of LBS services by endusers.

As usability is an important constraint, the research involved investigating whether a user-model can be constructed on the basis of context and interaction data. This model would need to drive an adaptivity engine to adjust the LBS system to a user's profile. This study aims to determine whether, on the basis of a user's context and interaction behaviour with an LBS system, the following characteristics about the user can be deduced:

- Computer proficiency

- Spatial literacy

- Experience in the usage of interactive applications

The expectation is that a highly proficient and experienced user will interact with the system in a significantly different manner to a user who considers themselves inexperienced, and that this difference can be measured. It is also expected that users of a similar skill level will interact in a comparable way. Less certain is whether the spatial literacy of a user will affect how the user interacts with an LBS application, and whether the difference between users of a different spatial literacy can be measured. A user's context, such as their choice of platform, software, and hardware, is expected to reflect users' preferences, which in turn are assumed to be in line with their skills and aptitudes. It is therefore predicted that contextual data will contribute to modelling the characteristics outlined above.

A prototype, named POMICOS, has been implemented on the basis of the designed framework to achieve four goals:

1. to demonstrate the feasibility of the design, and its effectiveness in addressing the constraints

2. to collect user interaction and context data to investigate the above user modelling objectives

3. to build a basic user-model using the collected data if the user-modelling objectives are achieved

4. to obtain feedback from users on the general concept

\subsection{Core Components}

A proposed high-level architecture of a conceptual LBS framework is outlined in. Fig. 2. It comprises a number of components for web mapping, user modelling, positioning and social interaction that are integrated via a synchronisation component. Users interact via a user interface component that is webbased. These components are detailed further in the following subsections. 


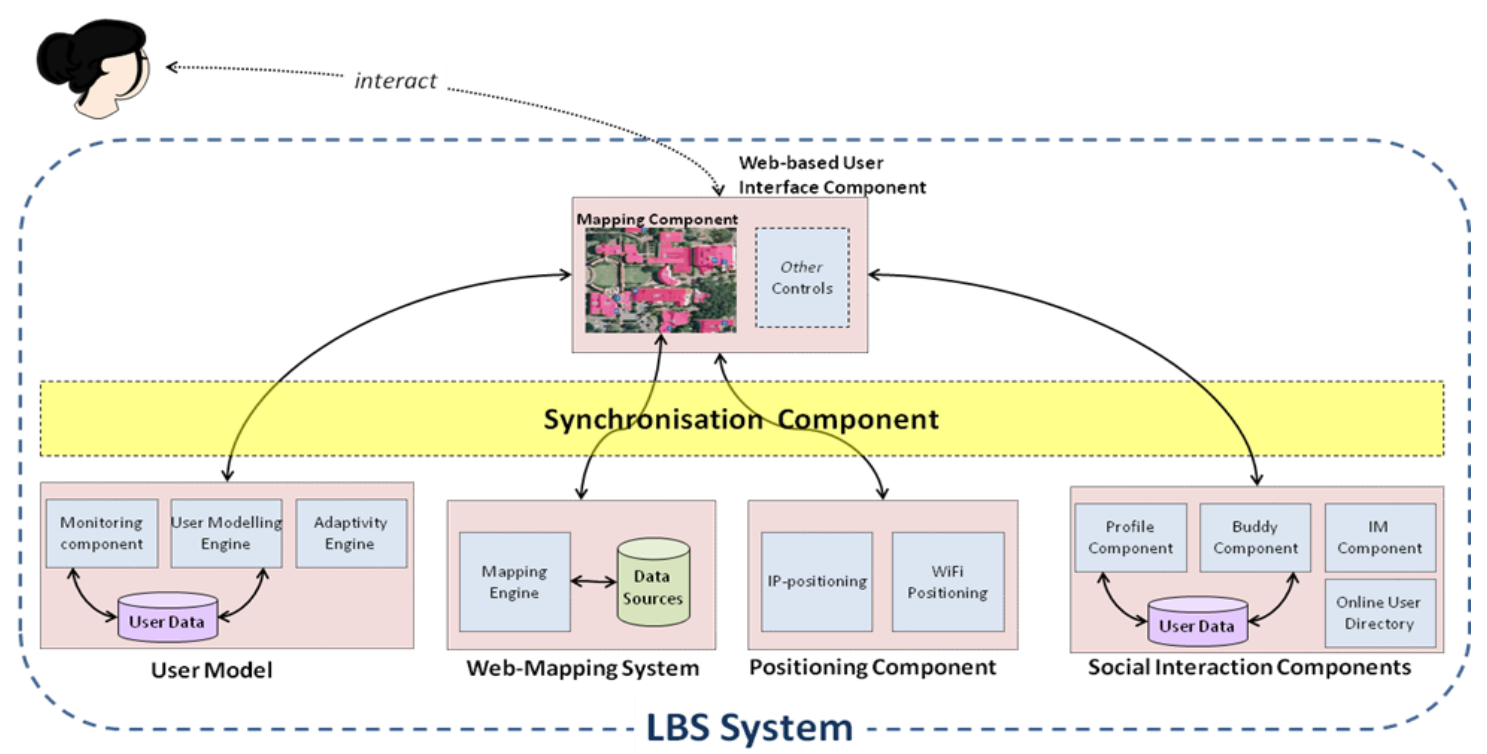

Fig. 2 : High-level architecture of conceptual LBS framework

\subsubsection{Web-based interface and mapping components}

As discussed in Section 2.2.2, offering applications as a web-based solution is a cost-efficient methodology, because it allows the use of existing web browsers and access to a global communications infrastructure, the Internet. In addition, a web-based solution can benefit from the relatively standardised protocols that exist to achieve interoperability. An embedded tile-based webmapping system was used as mapping component. This type of web-mapping system displays maps as a composite of tiled images. The browser displays the visible portion of this composite, while the invisible parts exist outside the viewable area, ready to appear if the user moves the map. This type of design is fast and intuitive, and highly suitable for server-side and client-side caching.

Communication between the client (browser) and the server should ideally be performed using AJAXbased methodologies [4], as traditional methods based on form submission are slow and interruptive. An AJAX-library was developed in JavaScript as part of this research, to manage the communication. To optimise screen real-estate and avoid overcrowding the interface, a tab-control was developed, allowing the user to switch between different content.

\subsubsection{Transparent multi-functional positioning component}

Because user location is paramount to an LBS system, any information available that can give an indication of the whereabouts of a user should be exploited to its fullest extent. This should preferably be done autonomously, without requiring user intervention, as it can degrade usability to specifically ask the user for input [34].

As outlined in Section 3.2.1, a web-based design was chosen for reasons of accessibility and cost, meaning that the positioning methods had to be limited to those typically available to a web-browser, and therefore to a computer.

Most systems rely on a single method to obtain a location [6, 19, 27]. However, multiple methods should be used in order to circumvent the limitations of availability and precision associated with different individual systems. For this reason, the positioning component was designed to include multiple methods. On initialisation, the component uses methods with a high availability, but typically a lower precision. If after initialisation it is determined that appropriate context information is available, the system will employ a method offering a higher precision. This hybrid design overcomes the constraint of availability, while optimising precision wherever possible. 


\section{WiFi-positioning}

Many computers, and most laptops, have access to a WiFi adapter. Triangulation-based WiFi positioning offers precisions of 13-40 metres, depending on network density [36]. Fingerprinting could offer higher precisions, from 2-3 metres for known access-point locations [22, 36], up to about 15 metres using access points of less precise locations [36, 63]. However, as fingerprinting requires a very extensive training dataset, it was not directly considered for this research. Although websites are not normally able to access hardware devices directly, it is possible to do so using a signed JAVA applet that runs inside the webpage. Technology to accomplish this was developed as part of the PlaceLab project [64]. The applet needs only to query the WiFi adapter to obtain the unique identifiers, MAC addresses, and signal strengths of the $\mathrm{WiFi}$ access points in range. A server-side look-up of position can then be performed in one or more databases that contain geographic coordinates for these MACaddresses. The location of the user can then be determined, for example by applying a weighted average of coordinate values based on signal strengths.

Access-point lookups may be performed online, for example using Wigle [65], an online repository of geocoded $\mathrm{WiFi}$ access points. Alternatively, lookups may be performed using local databases containing a regional extract of Wigle or another data-source, for performance reasons. It is also possible to manually determine the position of local wireless access points using georeferenced building maps or accurate GPS readings, which would offer increased accuracy compared to publicly sourced data. These lookup methods need not exclude each other. Rather, they complement each other in terms of coverage, accuracy, and performance.

\section{IP-positioning}

Another resource available to networked computers is the IP-address. There are a number of ways to obtain a location from an IP-address. The most basic approach is a look-up. There are many web-based applications on the internet that are able to provide global, city-scale locations for an IP-address, for example Host-IP [66].

Look-up based methods do not assume or exploit geographic proximity of network subnets, unlike cluster-based methods such as GeoCluster [25] and VRILS [39]. The Varying Resolution IP-Locating System (VRILS) was designed and implemented as part of this framework to provide an IP-positioning capability that tries to correlate network clusters to geographic clusters where possible. It was designed on the principle that computers on the same subnet are likely to be in geographic proximity. If evidence is found to the contrary, then a broader-scale geocode of a lesser spatial resolution is assigned to the network cluster, instead of declaring the cluster impossible to position accurately as GeoCluster does [25]. Precision is sacrificed to maintain accuracy, but only where necessary. The ideology behind VRILS is that an imprecise location is better than no location at all. VRILS can be configured to have any number of spatial precisions, although for the prototype implementation of this framework the geographic scales of "campus", "building", and "room" were used.

\section{GPS-based positioning}

GPS is the most widely known positioning methodology. The accuracy for handheld GPS is typically better than 13 metres, although sub-metre accuracies are possible using differential GPS [26], or using Continuously Operating Reference Station (CORS) networks [67, 68]. However, GPS typically does not work indoors or in highly built-up areas, due to the line-of-sight problem [26]. CORS networks overcome the problem of accuracy, but are expensive to establish, especially in areas of sparse population as is the case, for example, in Australia [67, 68]. Also, most computers do not have a GPS device, and if they did, a webpage in a browser would not easily be able to access the device. Although this barrier can technically be overcome, at this point in time only proposed solutions exist [69]. This limitation means that even on a GPS-enabled smart-phone, a web-page can not take advantage of the GPS capabilities. For these reasons, GPS-positioning was not included in the design of the framework.

\section{GSM positioning}

Positioning through cell-phone signals was also not included in the design of the framework. Although many laptop and phone devices possess the hardware to make it theoretically possible, as with GPS, there is a technical barrier to accessing this hardware from a web browser. But mostly, the typical precision of GSM positioning is reasonably poor, often between 100-200m at best [20, 23], making it unsuitable for usage in larger scale LBS applications. 
Error! Reference source not found. illustrates a comparison between various positioning methods and the spatial resolution of locations able to be obtained. Note how different WiFi-positioning methods are included, offering different levels of performance, coverage, or accuracy. Two IPpositioning methods are used, with VRILS offering potential accuracies varying from the size of a campus to the size of a room. For the prototype designed for this research, only some of the positioning techniques were included as indicated in the figure.

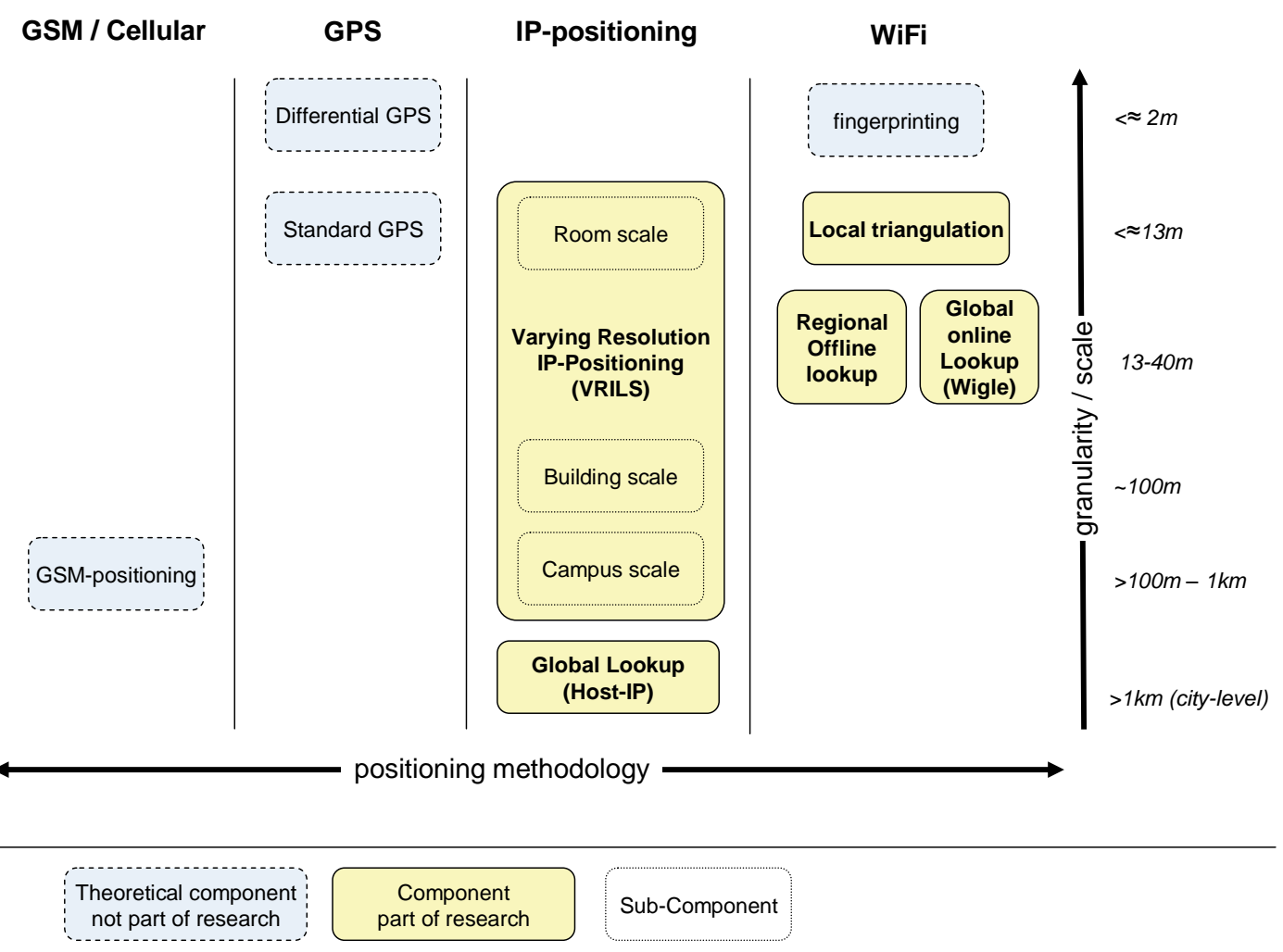

Fig. 3 Comparison of positioning methods and resolutions

\subsubsection{User modelling and adaptivity component}

As discussed in Section 2.2.5, adaptivity is a potential remedy for poor usability in complex systems. If a system adapts itself to the nature or the activities of the user, it is likely that the user's goals are more easily achieved. For example, a system could automatically display specialised tools related to what the user is doing, hide tools which are not needed, or suggest alternative or more relevant information. In order to drive the decisions of the adaptivity engine, a user model is needed that profiles the user in a qualitative and quantitative manner. Output from this user model would be used to drive the adaptivity engine.

The user model itself requires qualitative and quantitative input describing the user, and rules to transform this input into values that the adaptivity engine can use. As such, the framework needs to incorporate a user-monitoring component to collect contextual and interaction data describing the user. The rules of the user model need to reflect known relationships between monitored values and actual user characteristics. The user model can then provide user-customised instructions to the adaptivity component. For a user-model in an LBS system, these instructions could include:

- show or hide a tool or component

- display more or less information

- offer hints or tips

- focus on an inferred location of interest

- recommend a social contact 


\subsubsection{Social interaction component}

. It is not enough for a system to be capable of performing the tasks outlined in its specification, rather, a system must be attractive to its target users. Failing that, the uptake by users will be poor, and users will be quick to abandon it. Given the high usage by users of social networking sites, building in a social interaction linkage will potentially increase the attractiveness and uptake of LBS applications. Combining social interaction with the spatial dimension can also give rise to interesting new capabilities, such as the GeoLife2.0 friend-recommender system [70]. GeoLife2.0 recommends friends on the basis of common interests, as deduced from visited locations extracted from GPS logs. The recent popularity of social networking applications, such as Friendster [1], MySpace [3], Facebook [13], and Twitter [2] give a strong indication that social interaction is a major motivation for the use of applications.

To support social interaction and networking, the following capabilities are proposed to be supported:

- A user profile component, allowing users to share information about themselves

- An instant messaging component, to enable live communication

- A buddy component, to allow definition of relationships between users, enabling users to share their location and details with friends

- A directory of (online) users, to encourage people forming relationships

\subsubsection{Synchronisation component}

Most of the components that are part of the LBS framework require frequent and continuous interaction between the browser and the server. Examples of these interactions are:

- Send data from the monitoring component to the user model

- Check for changes to the status or location of buddies

- Check if instant messages have been received

- Refresh location based on new WiFi information

- $\quad$ Send message

If each component were to check for status updates independently, the amount and frequency of requests to-and-fro the server would be unacceptably high, and performance as a whole would suffer. For this reason, a synchronisation component was designed that communicates with the server to find out which components need updating. Only the components requiring a status update will then interact independently with the appropriate server-side components. The synchronisation component has a strongly coordinative role, and as such can help facilitate integration of heterogeneous components. Note in Fig. 2 how the synchronisation component controls the flow of data traffic between the client and the server.

The synchronisation component checks for updates at ever-increasing intervals, to minimise unnecessary traffic. Based on user activity, it decreases its polling interval to 1 second immediately after user interaction, allowing it to increase again to 5 second intervals during periods of inactivity. Whether components require an update is indicated by flags that are set and unset in the user session.

\subsection{Prototype Design}

The POMICOS prototype is a basic design and implementation of the proposed framework that follows the architecture described in Section 3.2. The purpose of this prototype is to provide a proof of concept and feasibility, as well as providing a platform for data collection in order to create rules for the user model. The user model and adaptivity engine were therefore not yet part of the prototype implementation, but a proposed user-model based on the outcomes is presented in section 4.3. It should be noted that all third party software used for the development of POMICOS was open source, demonstrating that cost-efficient technology, as described in Section 2.2.2, is an effective and feasible approach to addressing cost constraints.

A user monitoring component was incorporated in the prototype design to collect qualitative and quantitative information about the user. The qualitative parameters are mostly related to the user context and environment, whereas the quantitative parameters include the user of correction keys, and 
those factors of the Keystroke-level Model [71] that can be measured from inside a webpage. These include mouse-movement speed, typing speed, and hand-movement speed. The measurement of speed is performed by determining the average latency between subsequent actions. An action is deemed to have finished when a different action has commenced. For each action, cut-off times are put in place beyond which an action was deemed incomplete (and ignored). The cut-off times chosen are assumed to be well outside reasonable latencies. Hand- and mouse-movements are deemed incomplete beyond 3 seconds, and key presses have a maximum allowable latency of 1.5 seconds. Beyond 1.5 seconds, the key presses are assumed to belong to unrelated events. The number of inactive periods is also counted. An inactive period is a monitoring interval during which the user has not performed any actions.

The monitoring component sends the interaction data to the server at ever increasing intervals. The reason why intervals are increased is that it is assumed that the user will be more tired after a long period of interaction, and that the behaviour of the user becomes less representative of the user's true characteristics [72]. An alternative approach would be to adapt the duration between samples to the interaction behaviour of the user, with more samples taken during periods of increased activity.

To enable social functionalities, a basic design of the social interaction components listed in section 3.2.4 was included in the prototype. Although integration with existing social networking applications would be preferable for a production system, for reasons of control and flexibility it was decided to create custom capabilities for the prototype. To support these custom social networking components, it was necessary to accommodate for user accounts and authentication mechanisms in the design. To protect users' privacy, the ability to appear offline is offered, allowing users to temporarily stop sharing their location and online status.

Fig. 4 illustrates the flow of communication to and from the different components of POMICOS. As can be seen, the synchronisation component has client-side and server-side interfaces that communicate to update components on either side. Note that the user modelling component and the adaptivity component have not yet been developed and require further research and investigation. 


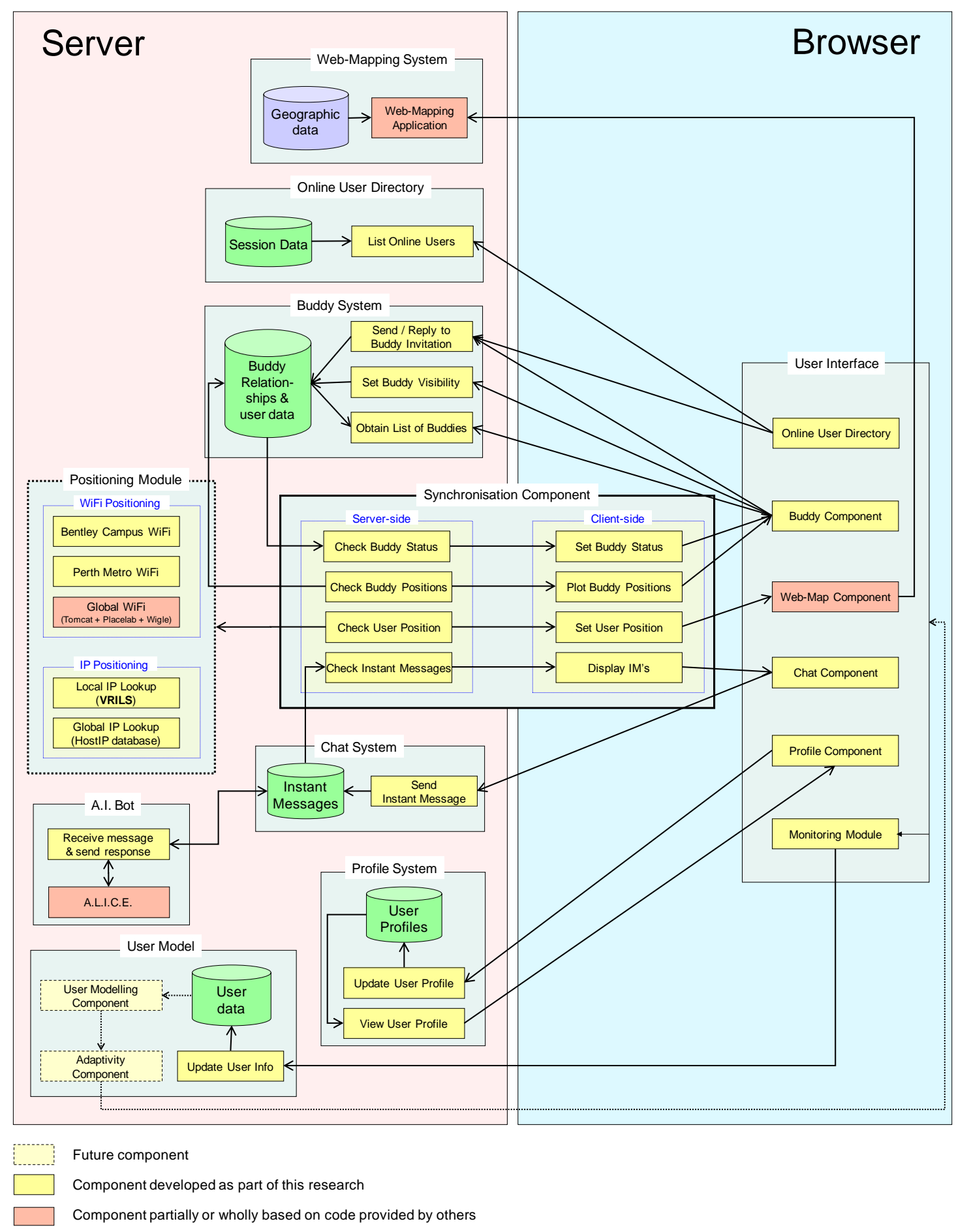

Fig. 4 POMICOS Architecture

\subsection{Prototype Implementation}

The components described in Section 3.2, as well as the encapsulating framework, were developed using the languages PHP, JSP, JAVA, JavaScript, and HTML.

The web-mapping component was populated with aerial photography, buildings, and facilities available on campus, such as cafes, toilets, and ATM's. For reasons of performance, cost and interoperability, the open-source web-mapping client Ka-Map [42] was chosen as client, which uses MapServer [41] as 
back-end. MapServer is able to consume WMS and WFS data based on OGC standards. However, for performance reasons only local data extracts were used.

Global positioning support was implemented using HostIP [66]. Local IP-positioning was implemented using VRILS, which was populated using information collected by volunteers [39].

WiFi-positioning was supported at global, regional (Perth metropolitan), and local scale. A JAVA applet using Placelab technology [64] was used to scan for wireless access points. Global WiFipositioning was performed through an online lookup in the Wigle online repository [65]. Regional WiFi triangulation was performed offline using a data extract obtained from Wigle. Local triangulation was supported by data collected on-campus; wireless access points were digitised from geo-referenced floor plans, and attributed with MAC addresses that were determined using a laptop. Fig. 5 shows the tab-sheet containing the positioning control, which displays the location of the user. The location is also plotted in the mapping component.

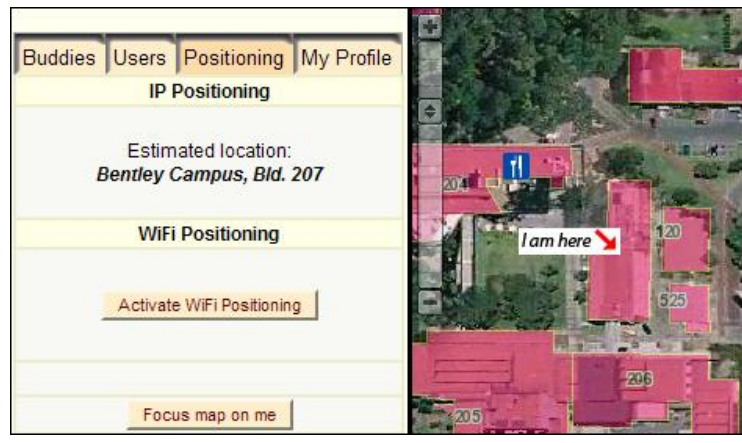

Fig. 5 Screenshot of the client-side positioning control

An Artificial Intelligence chat-bot was integrated into the social interaction components using scripts and content from the ALICE A.I. Foundation [73]. The chat-bot is available from instant messaging component, with the username "Alice". Users are able to chat to this bot when no other users are online. Although answers provided by this bot are typically generic and vague, it was proven to be quite entertaining. This feature was implemented mostly to ensure that volunteer users would be able to continuously and actively interact if few other users were online to chat with. Typing speed was considered a key metric for user monitoring as it is likely to reflect experience and proficiency, hence the importance of ensuring that users had the opportunity and motivation to use the keyboard.

All transactions are performed using cookies, allowing a certain degree of security to exist for social interaction, while keeping the security mechanism simple.

During the initial design phases, it was correctly anticipated that by the time of prototype implementation, mobile devices would have advanced enough to deal with rich web content like desktop browsers can. POMICOS was successfully tested on a Windows Mobile 6.0 PDA phone, running the Opera Mobile web-browser. With the exception of WiFi-positioning, which requires support for JAVA applets, all components worked correctly. Although screen real-estate will remain a problem on small devices, Opera deals with this by zooming-in and out on different parts of the rendered webpage, as can be seen in Fig. 6:
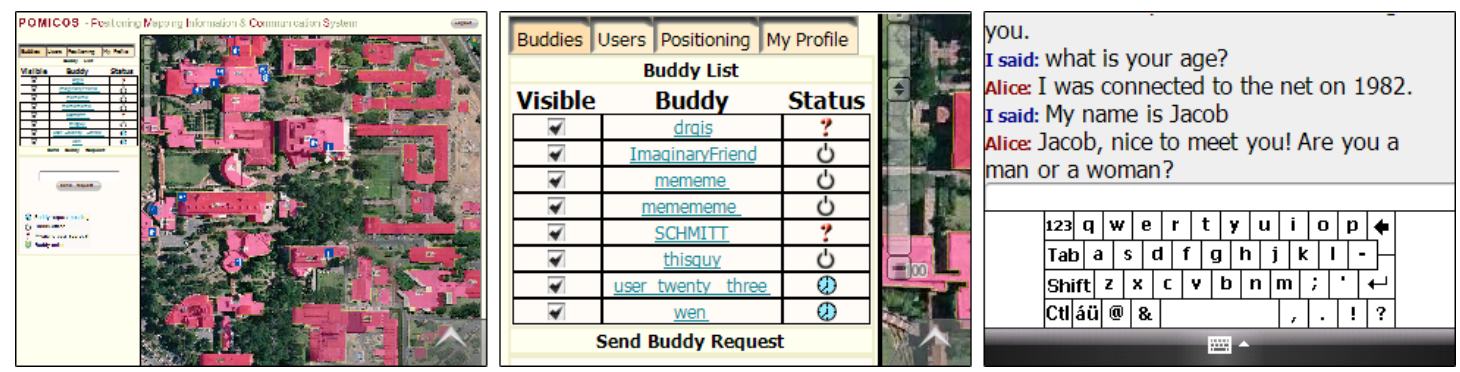

Fig. 6 Screenshots of POMICOS accessed from a PDA phone running Opera Mobile 


\subsection{Data Collection}

As explained in Section 3.1, this study aims to model user characteristics using contextual and interaction data, in order to drive adaptive changes to improve usability. Usage data had to be collected to identify and determine the nature of any relationships between the collected data and actual user characteristics, and to construct a user-model on the basis of the collected data.

As described in Section 3.2.3, POMICOS includes a monitoring component to capture interface events, and store contextual information about its users. A trial was conducted using volunteers, who were asked to create an account for POMICOS and use it for at least 20 minutes. This time span was chosen because it would result in at least 34 samples from the monitoring component. A longer time span could possibly discourage volunteers, whereas a shorter time span would result in less than 30 samples per user. Volunteers were given a sheet of instructions that would lead them through the core functionality of POMICOS, to ensure broad coverage of activities, and to engage them. Following this, the volunteers had to fill out an online questionnaire where users were asked to describe themselves in terms of skills, interests and experience with regards to spatial information, websites, and computer literacy. Answers in this questionnaire constituted the ground truth for the analysis to build the usermodel. All quantitative questions were on a scale between from 1 to 10 . Information on age-group and gender was also collected, as well as feedback on the overall experience. The volunteers were students and staff from various areas of Curtin University, recruited in various ways through several channels.

Table I shows the main data that was collected, measured, or requested in the questionnaire. This data includes all monitoring data that could be determined from the browser, as well as questionnaire answers reflecting background, experience, skill, and preferences.

Table I Variables determined for data collection

\begin{tabular}{|l|l|l|}
\hline User Context \& Environment & User-interaction & Questionnaire answers \\
\hline Last Location & Number of corrections (delete or & Computer proficiency \\
Colour depth of the screen & backspace) & Spatial literacy \\
Browser name & Number of key presses & Usage of interactive websites \\
Browser version & Number of hand movements between & Usage of chat applications \\
Horizontal screen resolution & keyboard and mouse & Fondness of browsing \\
Vertical screen resolution & Number of mouse-clicks & Fondness of interactive websites \\
Width of the browser window & Number of sample periods & Fondness of spatial information \\
Height of the browser window & Number of inactive periods (no events & Alias used in prototype \\
Operating system & during period) & How well it worked (technical \\
& Average key-press latency & issues) \\
& Average mouse-movement time & Did it resolve the user location \\
& Average hand-movement time & Website speed \\
& Number of Buddies & Did the user try WiFi positioning \\
& & Did WiFi positioning work \\
& & Age-group \\
\hline
\end{tabular}

The user session data was used to determine whether the user had used the system for a sufficient amount of time. If they had used the system for less than 20 minutes, a message would appear above the questionnaire warning that their participation would be in vain if they did not use the system for at least 20 minutes. After using POMICOS for a cumulative 20 minutes, it would issue a notice to the user that they were allowed to proceed to the questionnaire.

Volunteers could preserve their privacy if they wished; they had the ability to hide their location from buddies if they desired to do so, and were not obligated to create buddy relationships with other users. Users were not requested to provide personally identifying information.

\section{$4 \quad$ Results}

This section looks at the general feedback of volunteers from the survey, presents an overview of the collected data, and elaborates on the analysis of the collected user data. A basic user-model is then presented on the basis of the analysis results. Lastly, practical issues encountered during the research are further elaborated upon for the benefit of other researchers. 


\subsection{General Outcomes \& Feedback}

The data collection was conducted in early September 2008, and went without major problems. User feedback and usage records showed that the prototype performed reliably, and users made use of the available functionality as expected, and without problems. This outcome shows that some of the main objectives have already been addressed to at least some degree:

- The prototype implementation incorporated satisfactory positioning support, accommodating for both indoor and outdoor users; Positions were provided for $86 \%$ of the users, out of which $82 \%$ were at building-level precision. WiFi-positioning was supported in many parts of campus.

- Being web-based, the prototype exhibited a relatively high degree of integration, interoperability and standardisation, especially with the incorporation of an OGC-capable map component

- The prototype was developed without incurring expenses on software, hardware (other than an existing web-hosting platform), or positioning infrastructure, proving that the proposed framework can successfully circumvent cost as a constraint

The screenshot shown in Fig. 7 gives an impression of what POMICOS looked like during the data collection.

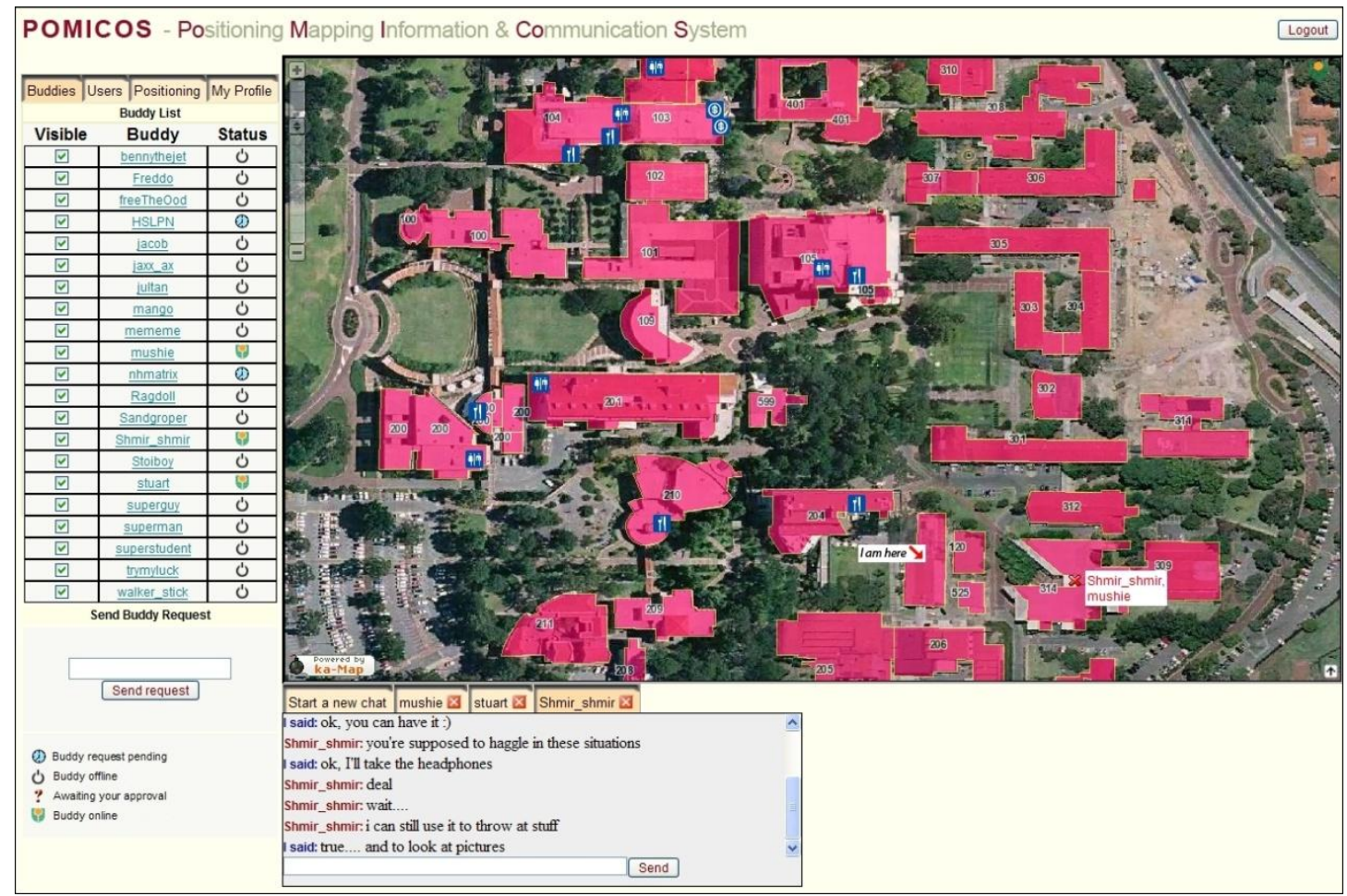

Fig. 7 Screenshot of the POMICOS interface during data collection

In total, 57 people have participated who used the system for the required amount of time, and who completed the questionnaire. An additional 9 people had tried to participate, but did not use the system for the required amount of time. The median number of samples recorded by the monitoring component for the 57 users during periods where they were active was 32, as the monitoring component did not measure interaction during idle periods. This corresponds to a median active usage time of at least 17.5 minutes. Because the polling interval of the monitoring component increases with time, and because idle periods are not counted, the exact total usage time could not be determined. But the active usage time varied between at least 4 minutes up to at least 12.5 hours. Of the 57 users, $32 \%$ were female, $68 \%$ were male. Participants were aged from 18 up to over 55, with the majority of 
participants (49\%) being between 18 and 23. Of all participants, 46 people had left feedback at the end of the questionnaire (which was optional).

The feedback regarding the system was generally positive, with most people indicating approval of the system in general; 14 people specifically indicated they enjoyed using the system, with one user specifically stating that the combination of functionality was good. Various users indicated they liked the automatic positioning, the chat component, and the concept of seeing where their friends are, which is something not offered by normal social networking applications. In particular the ability to chat to an artificial intelligence bot seemed to appeal to people. Some people indicated the performance of the map was slow, which was most likely during periods of higher load. Others also indicated that the positioning engine did not get their building quite right, which was mostly in cases where neighbouring buildings shared a single pool of IP-addresses among them.

From the feedback it showed that in total 8 people had some degree of trouble operating the map. Out of these 8 users, 7 gave themselves a rating for spatial literacy which was lower than that of the average user, and 1 rated slightly above the average. This is evidence that spatial literacy can be a constraint to the successful operation of LBS systems. It should be noted, however, that these users' ratings for fondness of spatial information and interactive websites did not demonstrate an adversity to either.

In terms of usability, the ratings on how well the application worked showed that the $20 \%$ of the users that gave ratings of 5 or below were found to struggle with the tabbed chat component or the interactive map functionality, judging by their comments. A few users got "lost" on the map, or thought a component malfunctioned because it did not behave as they expected. On the other hand, 2 users specifically stated that the application was easy to use or user friendly. These results show the variation in skill level of users, and support the need for the proposed user modelling and adaptivity components to help applications accommodate users of different skill levels and experience.

\subsection{Analysis Methodologies and Results}

Even though a large number of variables were collected, this analysis focuses on those key user characteristics that are considered most beneficial to model for adaptive web-based geospatial applications. These include computer proficiency, experience, and spatial literacy, and on the variables used to predict them.

Fig. 8 shows the histograms of the ground-truth for the key user characteristics. These histograms indicate the frequency of self-rated scores as provided by users in the questionnaire. Note that the distribution of computer proficiency matches the distribution for usage of interactive websites closely, showing peaks in the medium-high range, and few lower values. Spatial literacy shows a more even distribution, having more lower scores in comparison.
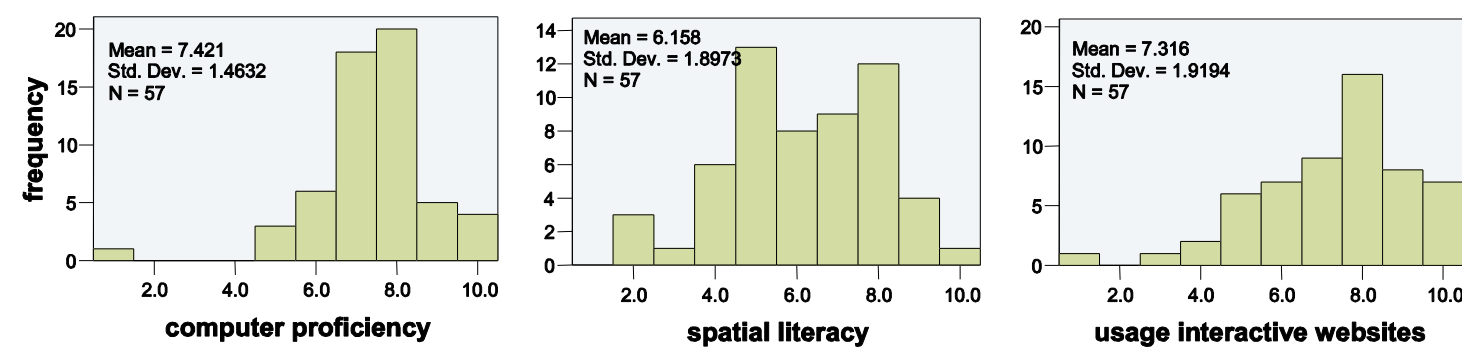

Fig. 8 Histograms of key questionnaire variables

To determine which predictive variables showed potential for significant relationships, a cross matrix of linear correlation coefficients was built up between the quantitative variables of the survey, and the quantitative variables measured by the monitoring component (see Table I). A direct linear correlation analysis between the survey results and the measured quantitative variables for all 57 records did not show any strong relationships, but weak relationships $(\mathrm{r} \approx 0.3)$ were found for the variables describing typing speed, mouse speed, and hand movement speed. As described in Section 3.2.3, these parameters were based on latencies between interface events. Histograms of these variables are shown in Fig. 9: 

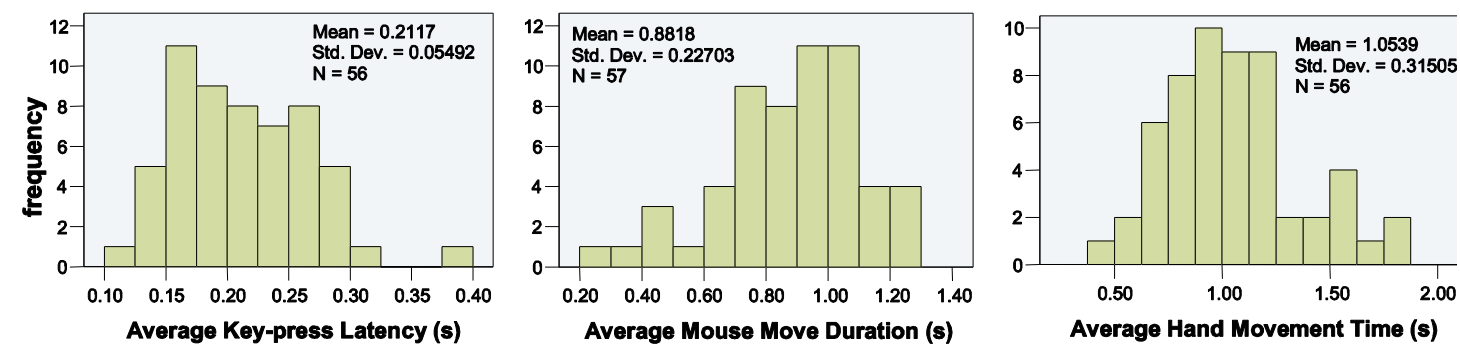

Fig. 9 Histograms of key user-monitoring variables

The user-monitoring component counted each monitoring interval during which the user had not performed an action. Based on this count, it could be seen that a substantial number of user records were based on a limited amount of interaction. In other words, although some users had used the system for sufficient time, they had been inactive for a significant proportion of it. Also, it was found that correlations within individual age groups, in particular age-groups 24-29 and 30-36 years old, were significantly higher compared to all age-groups combined. For this reason, the data used for further analysis was filtered down to include only these two age groups, and only those users that had been active during at least 26 monitoring intervals. This sub-sample comprised a total of 30 user records, who had been active during 26 to 143 monitoring intervals, with a median period of activity of at least 20 minutes. In this subsample, $70 \%$ were male and $30 \%$ female, with ages between 24-36.

It was found that relationships were typically non-linear. This is because the independent variables represented latencies, which become increasingly less likely to decrease as they become smaller. For this reason, a natural logarithm was applied to the variables prior to subjecting them for further analysis. After performing this transformation, the correlation coefficients were determined as shown in Table II.

Table II Linear correlation coefficients between key interaction parameters and user characteristics

\begin{tabular}{|l|c|c|c|}
\hline \multirow{2}{*}{ User Characteristic } & \multicolumn{2}{|c|}{ Natural Logarithm of Interaction Parameters } \\
\cline { 2 - 4 } & key-press latency & $\begin{array}{c}\text { mouse move } \\
\text { duration }\end{array}$ & $\begin{array}{c}\text { hand movement } \\
\text { time }\end{array}$ \\
\hline computer proficiency & -0.55 & 0.01 & 0.25 \\
\hline spatial literacy & 0.14 & -0.20 & 0.37 \\
\hline usage of interactive websites & -0.34 & -0.07 & 0.48 \\
\hline
\end{tabular}

These results show that people rating themselves as more computer proficient appear to type faster. This is in line with results found by Thomas et al. [59], who found a positive correlation between programming scores and typing speed for computer science students. It also shows that people rating themselves as more computer literate or experienced in interactive websites seem to take longer to move between keyboard and mouse. This can perhaps be explained by the fact that people who type faster can be expected to type blindly, keeping their eyes focussed on the screen, and taking slightly longer to find the mouse. People who type slower often do not type blindly, and therefore focus on their keyboard while typing, such that the mouse is within their vision while they switch. People who are more spatially literate seem to move the mouse at a higher speed, which may be because they are more used to graphical applications. People from various areas of spatial sciences may differ significantly in computer usage, which may explain the poor correlation between spatial literacy and key-press latency.

Different methods of analysis were used in order to find the most suitable way to allow users to be classified according to their interaction behaviour. As described in Section 2.2.6, user-models are often based on stereotyping, meaning that users are placed into classes according to certain qualitative and quantitative properties. For this reason, analysis using WEKA's [74] implementation of the decisiontree algorithm C4.5 [75] was performed. This method sorts records into nominal classes, based on numeric or nominal inputs. The inputs to the model were key-press latency, mouse move duration, and hand movement time. 
Although results were generally positive, it was found that the reported accuracy of the decision-tree analysis was significantly worse when performing a normal leave-one-out cross validation. This could largely be explained by the class-width being comparatively narrow, such that leaving out one record could off-set the classification enough for a record to narrowly miss its original class. An inspection of the confusion-matrix, showing predicted classes versus original classes, confirmed this. A small numeric lack of precision will result in a complete inaccuracy when numeric values are treated as nominal. For this reason, the percentage of records that were classified within one class-width of their original class is included in the results. Table III shows the percentage of correctly classified records for the key variables.

Table III Percentage of correctly classified records for C4.5 classifications

\begin{tabular}{|l|c|c|c|}
\hline \multirow{2}{*}{ Predicted User Characteristic } & \multicolumn{2}{|c|}{ percentage of correctly classified records } \\
\cline { 2 - 4 } & $\begin{array}{c}\text { without } \\
\text { cross-validation }\end{array}$ & $\begin{array}{c}\text { exact } \\
\text { leave-one-out } \\
\text { cross-validation }\end{array}$ & $\begin{array}{c}\text { leave-one-out } \\
\text { cross-validation, }+/-1 \\
\text { class }\end{array}$ \\
\hline computer proficiency & $80.0 \%$ & $23.3 \%$ & $76.7 \%$ \\
\hline spatial literacy & $70.0 \%$ & $13.3 \%$ & $40.0 \%$ \\
\hline usage of interactive websites & $73.3 \%$ & $13.3 \%$ & $40.0 \%$ \\
\hline
\end{tabular}

An attempt to merge classes did not help to improve the results, because the $\mathrm{C} 4.5$ algorithm became strongly biased towards the high-frequency classes.

Lastly, a multiple-regression analysis was performed, again using the natural logarithm of key-press latency, mouse move duration, and hand movement time as independent inputs. This resulted in the correlation coefficients shown in Table IV.

Table IV Prediction of user characteristics with interaction parameters by multiple regression

\begin{tabular}{|c|c|c|c|c|c|}
\hline \multirow[t]{2}{*}{ Modelled User Characteristic } & \multicolumn{3}{|c|}{$\begin{array}{c}\text { Interaction Parameters } \\
\text { (predictors) }\end{array}$} & \multirow[t]{2}{*}{$\begin{array}{l}\text { overall } \\
\text { p-value }\end{array}$} & \multirow{2}{*}{$\begin{array}{c}\text { Multiple } \\
\text { Correlation } \\
\text { Coefficient } \\
\text { (R) }\end{array}$} \\
\hline & $\begin{array}{l}\text { keypress } \\
\text { p-value }\end{array}$ & $\begin{array}{l}\text { mouse } \\
\text { p-value }\end{array}$ & $\begin{array}{c}\text { hand } \\
\text { p-value }\end{array}$ & & \\
\hline computer proficiency & 0.001 & 0.857 & 0.069 & 0.004 & 0.63 \\
\hline spatial literacy & 0.513 & 0.137 & 0.03 & 0.085 & 0.46 \\
\hline usage of interactive websites & 0.021 & 0.37 & 0.002 & 0.005 & 0.61 \\
\hline
\end{tabular}

The results show that relationships for computer proficiency and the usage of interactive websites are statistically significant, having small p-values. It also shows that the contribution to the models of mouse-movement time is generally not significant, with only a marginal contribution for the prediction of spatial literacy.

Despite the relationships for computer proficiency and usage of interactive websites being statistically significant, it was found that the multiple regression model performed comparatively poorly at reclassifying the data. Table $\mathrm{V}$ shows the number of correctly classified values, rounded to the nearest class:

Table V Percentage of correctly classified records for multiple regression classifications

\begin{tabular}{|l|c|c|}
\hline Predicted User Characteristic & \multicolumn{2}{|c|}{ percentage of correctly classified records } \\
\cline { 2 - 3 } & $\begin{array}{c}\text { without } \\
\text { cross-validation }\end{array}$ & $\begin{array}{c}\text { leave-one-out } \\
\text { cross-validation }\end{array}$ \\
\hline computer proficiency & $40.0 \%$ & $36.7 \%$ \\
\hline
\end{tabular}




\begin{tabular}{|l|l|l|}
\hline spatial literacy & $16.7 \%$ & $13.3 \%$ \\
\hline usage of interactive websites & $20.0 \%$ & $20.0 \%$ \\
\hline
\end{tabular}

The results show that the difference in accuracy between the cross-validation and the normal results are minimal. However, the accuracy as a whole is relatively poor in comparison to the $\mathrm{C} 4.5$ classification.

Although a significant amount of contextual data was collected, in particular related to the environment and the platform of the users, this data was found to be of minimal value for analysis, contrary to the expectations expressed in section 3.1. The reason was that most volunteers of the data collection participated from within buildings on campus, using computers that are set up in very similar ways by university IT departments. For this reason, the computer setup and environment did not reflect the characteristics, choices, preferences, or abilities of users, and as a result were not of value to building a user-model. It is expected, however, that contextual data related to the setup and environment of users would be of crucial value in situations where they are influenced and determined by personal user preferences, rather than corporate policies.

\subsection{Proposed User Model}

The purpose of the user-model is to drive adaptive changes to the system, and to adjust the behaviour of the system, in order to improve usability. Based on the results of the analysis, it would appear that a C4.5 classification is the most suitable basis for a user-model, as it performs best at reclassifying the data. Because of the strong correlations and relatively successful re-classification of data for computer proficiency, and because computer proficiency is likely to affect the perceived usability of a system, it was decided to base the user model on the decision tree of this variable. Because the contextual user data was too similar among users for modelling, no qualitative variables were incorporated into the user model. A diagram of the proposed user model is shown Fig. 10. Please note that the values have been converted from the natural logarithms that the decision-tree was based on, back to the actual monitored values.

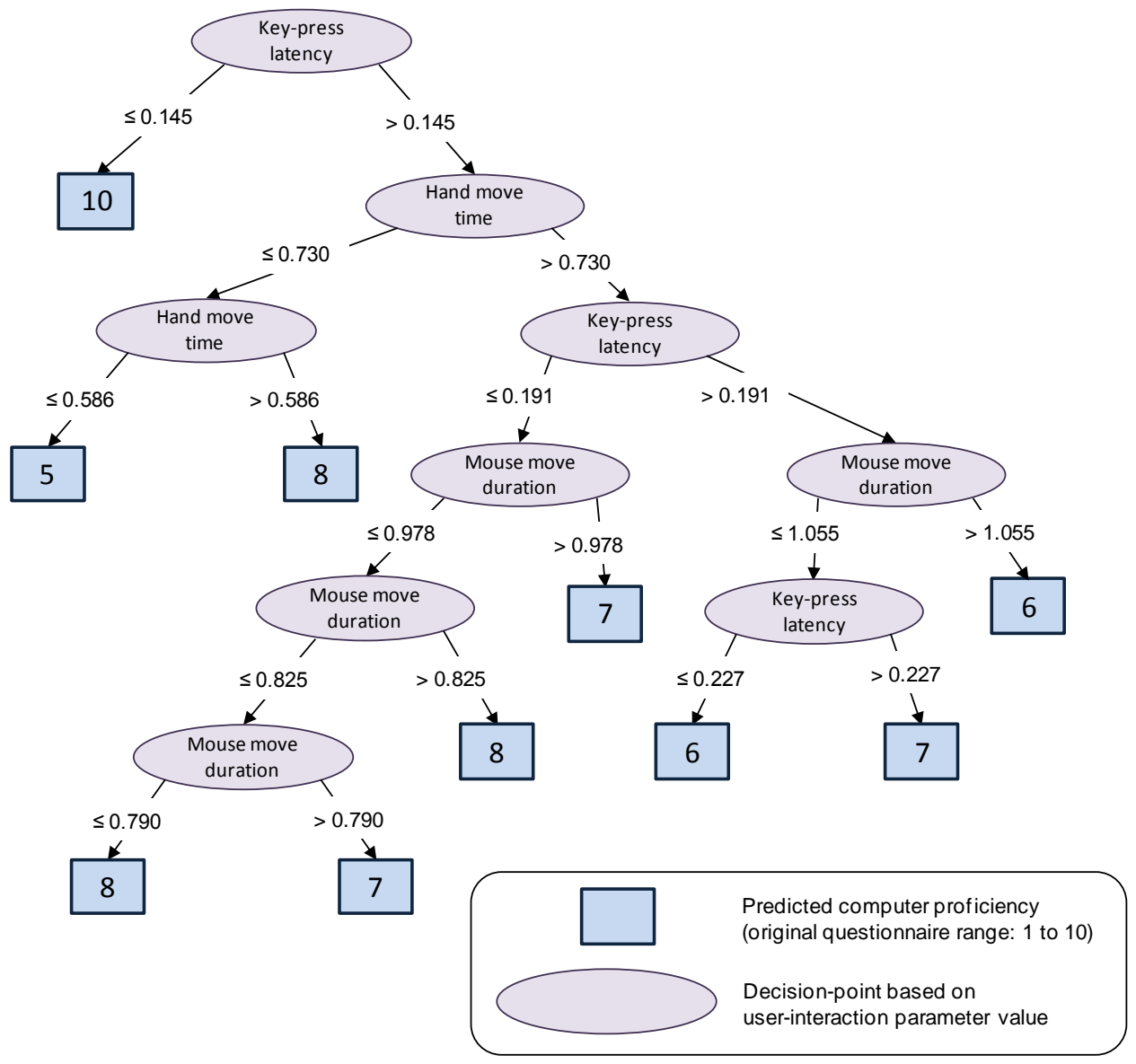


Fig. 10 Proposed user-model based on the C4.5 decision-tree of computer proficiency

The user-model can, with some degree of certainty, indicate how computer literate a user is likely to be, without having to ask the user. To estimate a user's computer proficiency, the decision-tree is followed using the interaction values applicable to the user, until a predicted rating is reached. For example, the first decision-point assigns users with a typing latency $\leq 0.145$ an immediate rating of 10 . In other words, users who can type faster than 414 keys per minute are assumed to be computer experts. Different combinations of values may result in a similar rating. The model shows how a user who types faster but moves the mouse slower may receive a rating of 7 , similar to that of a user who types slower but moves the mouse faster.

On the basis of predicted ratings for computer proficiency, information can be hidden or revealed, content of various levels of difficulty suggested, and functionality can be increased or simplified. If it is suspected that a user may have difficulties working out how to use a website, tips could be offered to guide the user through the functionality.

\subsection{Challenges Encountered}

This research project as a whole has covered a broad spectrum of fields and activities. As such, some unexpected problems were encountered, which are outlined below for the benefit of other researchers:

The WiFi access points used for this research broadcasted around 12 emulated MAC-addresses per single access point. These MAC-addresses could change if an access point was reset. For research in WiFi positioning using a business-network, it would be wise to investigate the nature of the wireless infrastructure first.

Although special firewall-rules were initially put in place to ensure the accessibility of the server, some internal firewalls which had undergone recent changes were overlooked, temporarily preventing access to potential volunteers from within the same building. It would be prudent to verify accessibility of a server from various locations prior to an online data collection exercise.

Despite instructions to volunteers being very clear and concise, and automated time-warnings being in place, $15 \%$ of volunteers did not use the system for enough time, or did not properly follow instructions. It cannot be emphasised enough how important proper instructions are.

It was particularly difficult to get enough volunteers, despite volunteers being entered into a draw for a popular multi-media device. It was found that advertising on public noticeboards was far less effective than personal promotion by people with credibility, such as lecturers.

\section{Conclusions}

Recent years have seen a strong increase of spatial technology into our daily lives. This research tries to highlight that despite the substantial amount of research being undertaken in LBS and ubiquitous spatial systems $[6,17]$, it appears that there are a number of core constraints that are not adequately addressed, preventing LBS from reaching its potential. These constraints include the lack of suitable positioning-technologies, implementation costs of LBS, integration problems between different technologies due to lack of standards, and the HCI-related problems of usability and user-objectives.

This research has put forward an LBS framework based on existing and new technologies to try to address these constraints. To address the problem of unavailability of positioning methodologies, the framework incorporates a hybrid positioning component that uses different forms of WiFi and IPpositioning to obtain users' locations. Integration and interoperability limitations are addressed by taking advantage of the highly standardised environment of the web, in combination with a mapping component supporting OGC standards. To improve usability in typically complex and information-rich environments, it is proposed here to incorporate an adaptivity component, which adjusts functionality and content to a user's inferred characteristics, such as their adeptness and experience in using the system. These user characteristics would be determined by a user-model, which obtains input data from a monitoring component collecting context and interaction data. The framework also includes social 
interaction components for the purpose of increasing the appeal to the general public, as social interaction is seen as a powerful means to leverage the appeal of LBS systems.

A prototype called POMICOS was developed on the basis of the proposed framework, to assess its effectiveness in addressing the described positioning, cost, and integration constraints. This prototype has demonstrated that a hybrid positioning approach can improve availability and quality of positioning, that LBS systems can be implemented without incurring major hardware or software expenses, and that a web-based platform with support for OGC protocols can facilitate integration and offer a high level of interoperability.

POMICOS was used to collect data to investigate whether relationships exist between a user's interaction and context on one side, and their general understanding and adeptness at using a system on the other. Data collected by the monitoring component was related to answers provided by the volunteers in a questionnaire used as ground-truth. Users were also invited to offer their opinion on the prototype.

The results showed that the quality of the monitoring data was significantly less for users that were largely inactive, and that correlations within age-groups were stronger. A potential explanation is that the youngest age-group may not have taken the data-collection too seriously, and that older age-groups were not as keen on the social interaction, resulting in reduced interaction data to work with.

The expectation was that users of different adeptness and/or experience would interact differently in a way that can be measured, and that interaction behaviour among similar users would be comparable. Analysis of active people in the age-groups 24-29 and 30-36 years old showed that significant correlations exist for computer proficiency and usage of interactive websites, when using key-press latency and hand-movement times as predictors. Correlations with spatial proficiency existed, but were not significant. When performing a cross-validation, it was found that multiple regression performed poorly at predicting the dependent variables, but that $\mathrm{C} 4.5$ was able to correctly classify computer proficiency within one class-width in $76.7 \%$ of the cases. Without tolerance, the reported accuracy was excessively poor, because the discrete $\mathrm{C} 4.5$ classification rejects any records that are not perfectly accurate, even if they are close. Merging classes did not achieve the same as applying tolerance, because the results became biased towards fuller classes.

Contrary to expectations, the contextual information collected about the environment of the user did not yield meaningful results in analysis, probably because most volunteers were using very similar systems in similar environments, such that this data did not reflect any characteristics specific to the user, in terms of preferences, background, or skills. Based on this outcome, it seems likely that context data would contribute more if collected from users' home computers, rather than a provided standardised environment.

The manner in which user-interaction was monitored was relatively simple, and can probably be improved upon. Had the monitoring been performed in a controlled HCI research environment, the results might have been better and more significant. However, it would not proof whether the same results can be reproduced through monitoring from a live production website in an ordinary environment, which is what this research tried to establish.

Despite the sample size being small, the results suggest that a $\mathrm{C} 4.5$ decision tree may be a good basis for a user-model. A basic user-model has been presented, which could be incorporated into an LBS system to attempt to assess computer proficiency of users. It should be noted that this user-model is based on a small number of samples, and is intended as a proof of concept. The decision-tree is based on interaction with the POMICOS system by users from a university environment, and might have to be adjusted if applied to a different interface and audience. Ideally, data collection should be performed among volunteers from strongly varying backgrounds to prevent any bias.

User-feedback indicated that volunteers generally liked the concept of automated positioning in combination with mapping and social interaction, with many describing the prototype as innovative and enjoyable to use. Although some users struggled with the functionality, other users found the application easy to use, which emphasises the variation in skill level of users and supports the need for adaption to individual users. Some people with below-average spatial literacy, but no adversity towards spatial information or interactive applications, struggled with using the interactive map. This 
demonstrates that lack of spatial literacy can indeed be a constraint to usability in LBS systems. The fondness that volunteers showed of talking to an artificial intelligence bot suggests that a bot configured to provide assistance may be a useful alternative to traditional written instructions and manuals, and is worth further investigation.

To improve on the results of the user modelling, it would be necessary to have a larger amount of volunteers. There may also be different ways in which interaction of a user can be monitored, other than through the metrics used in this research, or perhaps more intelligent ways in which monitoring data can be interpreted. However, the results seem to indicate that interaction data could be of significant benefit for adapting a system on-the-fly to a user's estimated skill-level, resulting in improved usability.

\section{Acknowledgements}

The authors would like to acknowledge the support of the volunteers who have participated in the data collection. The recruitment of volunteers was assisted by a number of staff and students, who helped in various ways to advertise the research. The authors would also like to acknowledge the significant efforts of IT staff at Curtin University to permit and enable the hosting of POMICOS within the university network.

\section{References}

[1] Abrams J.: Friendster. Mountain View, California. http://www.friendster.com (2002). Accessed 8 Jan 2009

[2] Dorsey J.: Twitter. Twitter, Inc, San Francisco , CA. http://twitter.com (2006). Accessed 20 Apr 2009

[3] eUniverse: MySpace. MySpace Inc., Beverley Hills, CA. http://www.myspace.com (2003). Accessed 8 Jan 2009

[4] Garrett J.J.: Ajax: A New Approach to Web Applications. Adaptive Path. http://www.adaptivepath.com/publications/essays/archives/000385.php (2005). Accessed February 18, 2005

[5] Li F.L., Xiaolei; Bian, Fuling: Autonomic LBS based on context: Preview. In: International Conference on Wireless Communications, Networking and Mobile Computing, 2007 (WiCom 2007). IEEE, Shanghai, China, pp. 3261-3264 (2007)

[6] Völkel T., Weber G.: Location-based and Personalized Information Services for Spas. In: Interact 2005. Rome, Italy (2005)

[7] Nokia: Ovi Maps. Nokia. http://maps.nokia.com/explore-services/ovi-maps (2006)

[8] Locatrix Communications: Uandme. http://locatrix.com/solutions/uandme/ (2006). Accessed 12 Jan 2010

[9] Microsoft Corporation: MapPoint. http://www.microsoft.com/mappoint (2000). Accessed 12 Jan 2010

[10] Phelan S.: Multimap. (1995). Accessed 12 Jan 2010

[11] Lowry E., Doppelt A., O'Donnell P.: Urbanspoon. Seattle, Washington, United States. http://www.urbanspoon.com (2006). Accessed 1 Feb 2010

[12] Google Inc: Google Maps. Google Inc. http://maps.google.com (2005). Accessed

[13] Zuckerberg M.: Facebook. Facebook Inc., Palo Alto, CA. http://www.facebook.com (2004). Accessed 8 Jan 2009

[14] Bilton N.: Facebook Will Allow Users to Share Location. The New York Times, New York, U.S. http://bits.blogs.nytimes.com/2010/03/09/facebook-will-allow-users-to-share-location/ (2010). Accessed 9 Mar 2010

[15] Haklay M., Jones C.: Usability and GIS - Why Your Boss Should Buy You a Larger Monitor. In: AGI GeoCommunity '08: Shaping a Changing World. Stratford-on-Avon, UK (2008)

[16] Meng Y., Malczewski J.: Usability evaluation for a web-based public participatory GIS: A case study in Canmore, Alberta. Cybergeo: European Journal of Geography, Article 483 (2009)

[17] Yu S., Spaccapietra S., Cullot N., Aufaure M.-A.: User Profiles in Location-based Services: Make Humans More Nomadic and Personalised. In: International Workshop on Next Generation Geospatial Information, NG2I 2003. Cambridge, USA (2003) 
[18] Oppermann R., Specht M., Jaceniak I., Huettenhain R.: Hippie: A Nomadic Information System. In: 5th ERCIM Workshop on "User Interfaces For All". Dagstuhl, Germany, pp. 251253 (1999)

[19] Köppen B., Bunningen A.v., Muthukrishnan K.: Wireless Campus LBS: Building CampusWide Location Based Services Based on WiFi Technology. In: E. Stefanakis, M.P. Peterson, C. Armenakis, and V. Delis (eds.) International Workshop on Geographic Hypermedia, pp. 399-408. Springer-Verlag, Berlin (2006)

[20] LaMarca A., Chawathe Y., Consolvo S., Hightower J., Smith I., Scott J., Sohn T., Howard J., Hughes J., Potter F., Tabert J., Powledge P., Borriello G., Schilit B.: Place Lab: Device Positioning Using Radio Beacons in the Wild. In: 3rd International Conference on Pervasive Computing. Munich, Germany, pp. 116-133 (2005)

[21] Schilit B.N., LaMarca A., Borriello G., Griswold W.G., McDonald D., Lazowska E., Balachandran A., Hong J., Iverson V.: Challenge: Ubiquitous Location-Aware Computing and the "Place Lab" Initiative. In: The First ACM International Workshop on Wireless Mobile Applications and Services on WLAN Hotspots (WMASH). San Diego, CA, pp. 29-35 (2003)

[22] Anne M., Crowley J.L., Devin V., Privat G.: Localisation intra-bâtiment multi-technologies: RFID, wifi et vision. In: 2nd French-speaking conference on Mobility and ubiquity computing. Grenoble, France, pp. 29-35 (2005)

[23] Bill R., Cap C., Kofahl M., Mundt T.: Indoor and Outdoor Positioning in Mobile Environments - a Review and some Investigations. Geographic Information Sciences 10, 91 98 (2004)

[24] Gilliéron P.-Y., Merminod B.: Personal Navigation System for Indoor Applications. In: 11th IAIN World Congress. Berlin, Germany (2003)

[25] Padmanabhan V.N., Subramanian L.: An Investigation of Geographic Mapping Techniques for Internet Hosts. In: Applications, technologies, architectures, and protocols for computer communications. ACM Press, San Diego, California, United States, pp. 173 - 185 (2001)

[26] Zogg J.-M.: GPS Basics. U-Blox, Thalwil, Switzerland (2002)

[27] Holtkamp B., Gartmann R., Han Y.: FLAME2008 - Personalized Web Services for the Olympic Games 2008 in Beijing. In: Echallenges 2003. Bologna, Italy (2003)

[28] Spaccapietra S., Al-Jadir L., Yu S.: Somebody, Sometime, Somewhere, Something. In: International Workshop on Ubiquitous Data Management. Tokyo, Japan, pp. 6-16 (2005)

[29] Burstein M.H., McDermott D.V.: Ontology Translation for Interoperability Among Semantic Web Services. AI Magazine 26, 71-83 (2005)

[30] Gal A., Modica G., Jamil H., Eyal A.: Automatic Ontology Matching Using Application Semantics. AI Magazine 26, 21-32 (2005)

[31] Yu S., Aufaure M.-A., Cullot N., Spaccapietra S.: Location-Based Spatial Modelling Using Ontology. In: 6th AGILE on Geographic Information Science - "The Science behind the Infrastructure". Lyon, France (2003)

[32] ISO (1998) 9241: Ergonomic requirements for office work with visual display terminals (VDTs) -- Part 11: Guidance on usability.

[33] Schimiguel J., Baranauskas M.C.C., Medeiros C.B.: Inspecting User Interface Quality in Web GIS Applications. In: VI Simpósio Brasileiro de GeoInformática. University of Campinas, Campos do Jordão, SP, Brasil (2004)

[34] Mackinnon L., Wilson M.: User Modelling for Information Retrieval from Multidatabases. In: 2nd ERCIM Workshop on 'User Interfaces for All'. Prague, Czech Republic, p. 9 (1996)

[35] Kim K., Summet J., Starner T., Ashbrook D., Kapade M., Essa I.: Localization and 3D Reconstruction of Urban Scenes Using GPS. In: 2008 IEEE International Conference on Wearable Computers. IEEE Computer Society, Pittsburgh, United States, pp. 11-14 (2008)

[36] Cheng Y.-C., Chawathe Y., LaMarca A., Krumm J.: Accuracy Characterization for Metropolitan-scale Wi-Fi Localization. In: 3rd International Conference On Mobile Systems, Applications And Services. Seattle, Washington, pp. 233 - 245 (2005)

[37] Martin A., Ionut C., Romit Roy C.: SurroundSense: mobile phone localization via ambience fingerprinting. In: Proceedings of the 15th annual international conference on Mobile computing and networking. ACM, Beijing, China, pp. 261-272 (2009)

[38] Sheng-Po K., Hsiao-Ju K., Yu-Chee T.: The Beacon Movement Detection Problem in Wireless Sensor Networks for Localization Applications. IEEE Transactions on Mobile Computing 8, 1326-1338 (2009)

[39] Delfos J., Tan T., Veenendaal B.: Scale-dependency in IP-based positioning of network clusters. Journal of Location Based Services 2, 3-23 (2008) 
[40] Weyn M., Schrooyen F.: A WiFi Assisted GPS Positioning Concept. In: The Third European Conference on the Use of Modern Information and Communication Technologies. Gent, Belgium (2008)

[41] Lime S.: UMN MapServer. University of Minnesota, Minnesota. http://mapserver.gis.umn.edu (1996)

[42] Spencer P.: Ka-Map. DM Solutions Group, Ottawa, Canada. http://ka-map.maptools.org (2004)

[43] Refractions: PostGIS. Refractions Research, Victoria, British Columbia, Canada. (2000)

[44] MetaCarta: OpenLayers. MetaCarta, Cambridge, MA, USA. http://openlayers.org/ (2005)

[45] Teng C.-M., Chu H.-h., Hsu J.Y.-j.: Making Use of Serendipity: A New Direction for Pervasive Computing from a Sociological View. In: 2nd International Conference on Pervasive Computing (Pervasive 2004). Linz/Vienna, Austria, pp. pp. 303-308 (2004)

[46] Mabrouk M. (2008) OpenGIS Location Services (OpenLS): Core Services. 1.2 ed United States: Open Geospatial Consortium Inc., p. 169

[47] Goldberg D.W., Wilson J.P., Knoblock C.A.: From Text to Geographic Coordinates: The Current State of Geocoding. Urban and Regional Information Systems Association Journal 19, 33-41 (2007)

[48] Patterson L.: Department of Spatial Sciences. Curtin University of Technology. http://www.spatial.curtin.edu.au/ (2009). Accessed 5 May 2009

[49] Papatheodorou C.: Machine Learning in User Modeling. In: Machine Learning and Its Applications. pp. 286-294 (2001)

[50] Reichenbacher T.: Adaptive Methods for Mobile Cartography. In: 21st International Cartographic Conference (ICC). Durban, South Africa, pp. 1311-1322 (2003)

[51] Samulowitz M.: Designing a Hierarchy of User Models for Context-Aware Applications. In: Situated Interaction in Ubiquitous Computing - Workshop at CHI2000. The Hague, Netherlands (2000)

[52] Sashima A., Kurumatani K.: Seamless Context-Aware Information Assists Based on Multiagent Cooperation. In: The second International Workshop on Agent-based Approaches in Economic and Social Complex Systems (AESCS'02). Tokyo, pp. 39-46 (2002)

[53] Flesca S.: Mining user preferences, page content and usage to personalize Website navigation. World Wide Web 8, 317-345 (2005)

[54] Klaus-Dieter S., Bernhard T., Qing W.: Customising Web Information Systems According to User Preferences. World Wide Web 12, 27-50 (2009)

[55] Calero C.: An Applicable Data Quality Model for Web Portal Data Consumers. World Wide Web 11, 465-484 (2008)

[56] Chepegin V., Aroyo L., Bra P.D., Heckmann D.: User Modeling for Modular Adaptive Hypermedia. In: Workshop on Applications of Semantic Web Technologies for Adaptive Educational Hypermedia (SW-EL@AH’04). Eindhoven, The Netherlands (2004)

[57] Stephanidis C., Paramythis A., Sfyrakis M., Stergiou A., Maou N., A. Leventis, Paparoulis G., Karagiannidis C.: Adaptable and Adaptive User Interfaces for Disabled Users in the AVANTI project. In: Proceedings of the 5th International Conference on Intelligence and Services in Networks (IS\&N '98): Technology for Ubiquitous Telecom Services. Antwerp, Belgium, pp. 153 - 166 (1998)

[58] Hilbert D.M., Redmiles D.F.: Extracting Usability Information from User Interface Events. ACM Computing Surveys (CSUR) 32, 384 - 421 (2000)

[59] Thomas R.C., Karahasanovic A., Kennedy G.E.: An Investigation into Keystroke Latency Metrics as an Indicator of Programming Performance. In: Seventh Australasian Computing Education Conference (ACE2005). Australian Computer Society, Inc., Newcastle, New South Wales, Australia, pp. 127 - 134 (2005)

[60] Zhang J., Ghorbani A.A.: GUMSAWS: A Generic User Modeling Server for Adaptive Web Systems. In: Conference on Communication Networks \& Services Research (CNSR 2007). Fredericton, Canada, pp. 117-124 (2007)

[61] Yang Y., Aufaure M.-A., Claramunt C.: Towards a DL-based semantic user model for web personalization. In: Third International Conference on Autonomic and Autonomous Systems. Athens, Greece, p. 61 (2007)

[62] Peng X., Silver D.L.: Interface Adaptation Based on User Expectation. In: 21st International Conference on Advanced Information Networking and Applications Workshops 2007 (AINAW '07). IEEE Computer Society, Niagara Falls, ON, Canada, pp. 264-269 (2007) 
[63] Letchner J., Fox D., LaMarca A.: Large Scale Localization from Wireless Signal Strength. In: National Conference on Artificial Intelligence (AAAI 2005). Pittsburgh, Pennsylvania, pp. 15$20(2005)$

[64] Intel: Place Lab. Seattle. http://www.placelab.org (2004). Accessed 1 Aug 2007

[65] Wigle: Wireless Geographic Logging Engine - Plotting WiFi on Maps. http://wigle.net. http://www.wigle.net (2007). Accessed 1 Aug 2007

[66] Gornall S.: HostIP. "hostip.info". http://www.hostip.info (2005)

[67] Gordini C., Kealy A.N., Grgich P.M., Hale M.J., Gordini C.: Testing and Evaluation of a GPS CORS Network for Real Time Centimetric Positioning - The Victoria GPSnet ${ }^{\mathrm{TM}}$. In: Proceedings of the IGNSS2006 Symposium. Gold Coast, Australia (2006)

[68] Zhang K., Wu F., Wu S., Rizos C., Lim S., Roberts C., Ge L., Kealy A.: The Latest Development of a State-wide GNSS Network-based RTK System in Australia. In: International GNSS Conference. Sydney (2007)

[69] Chen S.-I., Kao F.-C.: The Design of Embedded GPS Navigation System Based on Internet Structure. In: 3th International Workshop on Positioning, Navigation and Communication (WPNC '06). Shaker Verlag, Hannover, Germany, pp. 97-103 (2006)

[70] Yu Z., Yukun C., Xing X., Wei-Ying M.: GeoLife2.0: A Location-Based Social Networking Service. In: Proceedings of the 2009 Tenth International Conference on Mobile Data Management: Systems, Services and Middleware - Volume 00. IEEE Computer Society, pp. 357-358 (2009)

[71] Kieras D.: Using the Keystroke-Level Model to Estimate Execution Times. University of Michigan, Michigan (2001)

[72] Ardissono L., Torasso P.: Dynamic user modeling in a Web store shell. In: 14th European Conference on Artificial Intelligence. Berlin, Germany, pp. 621-625 (2000)

[73] Wallace R.S.: A.L.I.C.E. ALICE A. I. Foundation, Oakland, CA, United States. http://alicebot.blogspot.com (2001). Accessed 1 Jun 2007

[74] Witten I.H., Frank E.: Data Mining: Practical machine learning tools and techniques. Morgan Kaufmann, San Francisco (2005)

[75] Quinlan J.R.: C4.5: programs for machine learning. Morgan Kaufmann Publishers Inc., Burlington, MA (1993) 